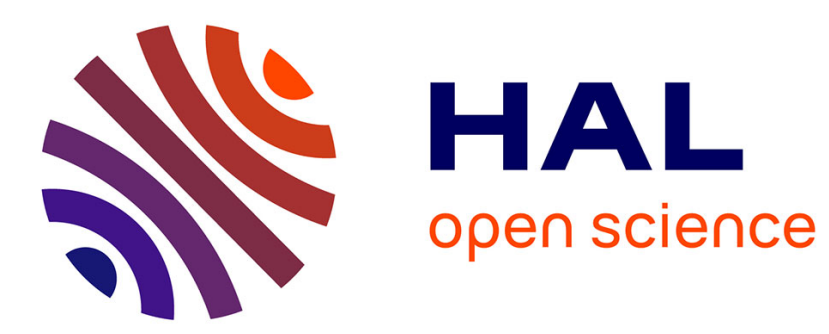

\title{
A coupling method for stochastic continuum models at diff erent scales
}

Yves Le Guennec, Régis Cottereau, Didier Clouteau, Christian Soize

\section{To cite this version:}

Yves Le Guennec, Régis Cottereau, Didier Clouteau, Christian Soize. A coupling method for stochastic continuum models at diff erent scales. Probabilistic Engineering Mechanics, 2014, 37 (-), pp.138-147. 10.1016/j.probengmech.2013.10.005 . hal-00940047

\section{HAL Id: hal-00940047 https://hal.science/hal-00940047}

Submitted on 31 Jan 2014

HAL is a multi-disciplinary open access archive for the deposit and dissemination of scientific research documents, whether they are published or not. The documents may come from teaching and research institutions in France or abroad, or from public or private research centers.
L'archive ouverte pluridisciplinaire HAL, est destinée au dépôt et à la diffusion de documents scientifiques de niveau recherche, publiés ou non, émanant des établissements d'enseignement et de recherche français ou étrangers, des laboratoires publics ou privés. 


\title{
A coupling method for stochastic continuum models at different scales
}

\author{
Y. Le Guennec ${ }^{\mathrm{a}}$, R. Cottereau ${ }^{\mathrm{a}, *}$, D. Clouteau ${ }^{\mathrm{a}}$, C. Soize ${ }^{\mathrm{b}}$ \\ ${ }^{a}$ École Centrale Paris, Laboratoire MSSMat, UMR 8579 CNRS, grande voie des vignes, 92295 Châtenay-Malabry, France \\ ${ }^{b}$ Université Paris Est, Laboratoire Modélisation et Simulation Multi-Echelle, MSME UMR 8208 CNRS, 5 bd Descartes, 77454 Marne-la-Vallée, \\ France
}

\begin{abstract}
In this paper, we present a novel approach that allows to couple two stochastic continuum models describing the same random medium at different observation scales. The coupling strategy is performed in the Arlequin framework, which is based on a volume coupling and a partition of the energy. Suitable functional space and coupling operator are chosen for the weak enforcement of the continuity between the two models. This choice ensures that the resulting mixed problem is well posed. The Monte-Carlo based numerical strategy for the solution of the mixed problem is briefly outlined. An application is presented, emphasizing on the interest of the chosen coupling operator. Finally, some remarks are provided concerning a stochastic multi-model coupling.
\end{abstract}

Keywords: Multiscale modeling, Coupling numerical method, Stochastic mechanics, Arlequin method

\section{Introduction}

Classical deterministic models provide global predictions that are satisfactory for many industrial applications. However, when one is interested in a very localized behavior or quantity, or when multiscale phenomena come into play, these models may not be sufficient. For instance the limited heterogeneity of a material modeled as a continuum may not have significant influence on its behavior at a large scale while it may influence greatly a local stress factor, which would typically be a fundamental quantity of interest for structural design. Unfortunately, for these problems, the information necessary to parametrize the relevant, very complex, models is usually not available. Stochastic methods have therefore been proposed $[1,2,3,4,5,6]$ and now appear unavoidable in multiscale modeling. Although the use of stochastic models and methods has expanded rapidly in the last decades, the related numerical costs are still often prohibitive. Hence, the application of these methods in a complex or industrial context remains limited. An important field of research is therefore concerned with the general reduction of the costs associated with the use of stochastic methods, for example by using iterative methods and preconditioners specially adapted to the structure of the

\footnotetext{
* Corresponding author

Email address: regis . cottereau@ecp.fr (R. Cottereau)
}

matrices arising in the Stochastic Finite Element (FE) method $[7,8,9,10,11]$, using reduced bases for the representation of random fields [12, 13, 14], domain decomposition $[15,16]$, or multigrid techniques [17].

Another approach consists, when it is possible, in trying to localize the use of stochastic models to limited regions of the computational domain, and to model the rest of the domain with a deterministic model [18, 19]. This is typically possible only when a homogenized medium can indeed be defined. The homogenized model is then used for the bulk of the domain, and the stochastic model is used close to boundaries, loadings or geometrical features that preclude the use of homogenized models, and in areas over which the quantities of interest are defined. Note that the areas over which the model should be stochastic are not necessarily obvious, although error estimation can help in that matter [20]. When it works, this approach can be seen as a model reduction technique, as the model is simplified wherever the simplification does not impact the quality of the estimation of the quantities of interest. It is appealing in terms of numerical cost for the direct solution of the corresponding problems, and also in terms of parameterization when the identification of the parameters of the models is considered.

There are unfortunately cases when homogenization theory cannot be applied straightforwardly, such as close to boundaries. This means in particular that over 
elongated domains, or when parameters fluctuate slowly with respect to the size of the domain, homogenization cannot be performed in the classical sense. However, in such cases, it might still be possible to perform a similar but more limited type of model reduction, and to partially upscale the parameters fields. A stochastic parameter fluctuating rapidly would hence be replaced by a parameter fluctuating more slowly over some parts of the computational domain rather than by a deterministic fully homogenized parameter.

This is the approach followed in this paper, and it requires two ingredients: (i) a recipe for upscaling partially a random medium, and (ii) a numerical method for coupling stochastic models described at two different scales. We consider that the models at the two scales are stochastic, in the sense that they are driven by partial differential equations with stochastic parameter fields. The first model describes the material heterogeneity at a micro scale, with a parameter field typically oscillating over small distances. The second model is coarser, and describes the same material at a meso-scale, typically with smaller variations in both amplitude and wavelength. The parameter of the second model may be seen as a filtered (or partially homogenized) version of the parameter of the first model. We will consider a case in which the upscaling method is known (step (i) above), so that the micro-scale and meso-scale (partially upscaled) models are given. This paper concentrates on the proposal of a coupling method for two stochastic models (step (ii) above).

Note that recent papers on stochastic multiscale mechanics have rather focused on an integrated approach to the problems (i) and (ii) seen above. Extensions of the multiscale finite element method [21] and variational multiscale method [22, 23] have been proposed for stochastic elliptic problems [24, 25, 26, 27] and for stochastic mixed formulations [28, 29, 30]. In these extensions, the authors discuss in particular the way to compute the meso-scale functional bases in efficient ways, using hypotheses of weak randomness [31, 32], interpolation [33] or stochastic collocation [26, 34, 35]. Extensions of the heterogeneous multiscale method were also proposed [36, 37], and the asymptotic behavior of the extensions of both the multiscale finite element method and heterogeneous multiscale method are discussed in [38].

Besides considering a segregated approach of (i) and (ii), the present proposal differs from the papers above in its focus. Indeed, the quantities of interest that we aim at approximating are assumed to depend on the micro-scale properties only locally. When this assumption is appropriate, the bulk of the model can be rep- resented by a meso-scale model without degrading the evaluation of the quantity of interest, and the zone where the micro-scale is considered can be seen as a zoom in a global meso-scale model. This paper therefore stands in the line of classical adaptive mesh and local enrichment techniques [39, 40, 41, 42], global-local iterative methods [43, 44, 45] and bridging methods [46, 47, 48, 49], widely developed for deterministic applications.

In particular, we construct our coupling technique in the Arlequin framework [46], which is a bridging method. Within this framework, the main issue of the paper lies in the choice of coupling operator and spaces that ensure that the resulting mixed formulation is well posed and that the results obtained from the coupled method are representative of the full micro-scale solution. This paper describes an extension of [50, 18], which considered the coupling of a deterministic model with a stochastic one. It bears similarities also with the paper [51] on discrete-to-continuum coupling, but the coupling operator is there completely different.

In Section 2, the macro-scale and meso-scale models of the random medium are described. In Section 3, the Arlequin modeling is introduced in the particular case of the coupling of two stochastic models. In Section 4 the numerical implementation is considered, based on the Monte-Carlo approach. In Section 5, some examples are provided. Finally, some conclusions are drawn in Section 6 and the extension of this method for two models driven by different partial differential equations (3D-beam coupling, for instance) is outlined.

\section{Stochastic modeling and upscaling}

In this section, we introduce the two stochastic continuum models that we will consider in the paper. We start with the micro-scale one. The meso-scale model is then obtained by considering a partial homogenization of the micro-scale mechanical parameter field. The micro-scale model is the reference one, and we only expect that the meso-scale solution can reproduce some of the features of the micro-scale solution. Finally, we will discuss at the end of this section the link between the partial upscaling considered here and classical homogenization.

\subsection{Micro-scale (reference) model}

Let us consider a domain $\Omega$ of $\mathbb{R}^{d}$, with outgoing normal vector $n$ and smooth boundary $\partial \Omega$, separated into Dirichlet and Neumann boundaries $\Gamma_{D}$ and $\Gamma_{N}$, such that $\Gamma_{D} \cup \Gamma_{N}=\partial \Omega, \Gamma_{D} \cap \Gamma_{N}=\emptyset$, and $\Gamma_{D} \neq \emptyset$. We consider 
Poisson's equation, with a random fluctuating mechanical parameter $k_{m}(x)$. In the particular case of homogeneous Dirichlet boundary conditions, the weak formulation of the corresponding stochastic boundary value problem reads: find $u_{m} \in \mathcal{V}_{m}$ such that

$$
a_{m}\left(u_{m}, v\right)=f(v), \forall v \in \mathcal{V}_{m},
$$

where $a_{m}: \mathcal{V}_{m} \times \mathcal{V}_{m} \rightarrow \mathbb{R}$ and $f: \mathcal{V}_{m} \rightarrow \mathbb{R}$ are defined, respectively, by $a_{m}(u, v)=\mathrm{E}\left[\int_{\Omega} k_{m}(x) \nabla u \cdot \nabla v d x\right]$, $f(v)=\int_{\Omega} f \mathrm{E}[v] d x+\int_{\Gamma_{N}} g \mathrm{E}[v] d x$, and $\mathrm{E}[\cdot]=\int_{\Theta} \cdot d P$ denotes the mathematical expectation. The mechanical parameter $k_{m}(x)$ is modeled as a positive, second-order, mean-square continuous stochastic field indexed on $\mathbb{R}^{d}$, and defined on a probability space $(\Theta, \mathcal{F}, P)$. We assume that it is statistically homogeneous and bounded almost surely for any $x \in \Omega c_{m}<k(x)<c_{M}$, with $c_{m}>0$. The functional space is $\mathcal{V}_{m}=\mathcal{L}^{2}\left(\Theta, \mathcal{H}_{0}^{1}\right)$, with $\mathcal{H}_{0}^{1}=\left\{v \in \mathcal{H}^{1}(\Omega), v_{\mid \Gamma_{D}}=0\right\}$. Endowed with the appropriate inner product and norm, $\mathcal{V}_{m}$ is a Hilbert space. Using Lax-Milgram theorem, it can be proved that the problem (1) has a unique solution $u_{m}$ (see for instance [52]). An approximation of that solution can then be obtained, for example, by using a Stochastic FE method [53, 54] or a Monte Carlo approach [55].

The field $k_{m}(x)$ will be called throughout micro-scale mechanical parameter field, the boundary value problem (1) will be called micro-scale mono-model problem, and its solution $u_{m}(x)$ will be called micro-scale monomodel solution. Note that we use the term mono-model for the problem and its solution, in order not to mistake them with the quantities relevant to the coupled system that will be introduced further down, and that will include a micro-scale component.

The micro-scale mechanical parameter field $k_{m}(x)$ is fluctuating, and those fluctuations are controlled by its power spectral density $\hat{R}_{m}(\zeta)$ (PSD), which is the Fourier transform in $\mathbb{R}^{d}$ of its autocovariance $R_{m}(y)=$ $\mathrm{E}\left[\left(k_{m}(x)-\underline{k}_{m}\right)\left(k_{m}(x+y)-\underline{k}_{m}\right)\right]$, where $\underline{k}_{m}=\mathrm{E}\left[k_{m}(x)\right]$ is the expectation of $k_{m}(x)$, and we assume that the auto covariance is integrable on $\mathbb{R}^{d}$. Although the power spectral density and autocovariance in general depend on the considered direction, we assume here that they are isotropic, so that they only depend on the amplitude of the $y$ and $\zeta$. Furthermore, we assume that a correlation length $\ell_{m}$ can be defined as $\ell_{m}=\int_{\mathbb{R}}\left|R_{m}(y)\right| d y / \sigma_{m}^{2}$, where $\sigma_{m}^{2}=R_{m}(0)$ is the variance of $k_{m}$. Examples of realizations of micro-scale parameter fields can be observed in Figures 1 (dashed lines) and 2 (upper figure), respectively in $1 \mathrm{D}$ and $2 \mathrm{D}$, along with an example of a triangular PSD in Figure 1 (upper figure).
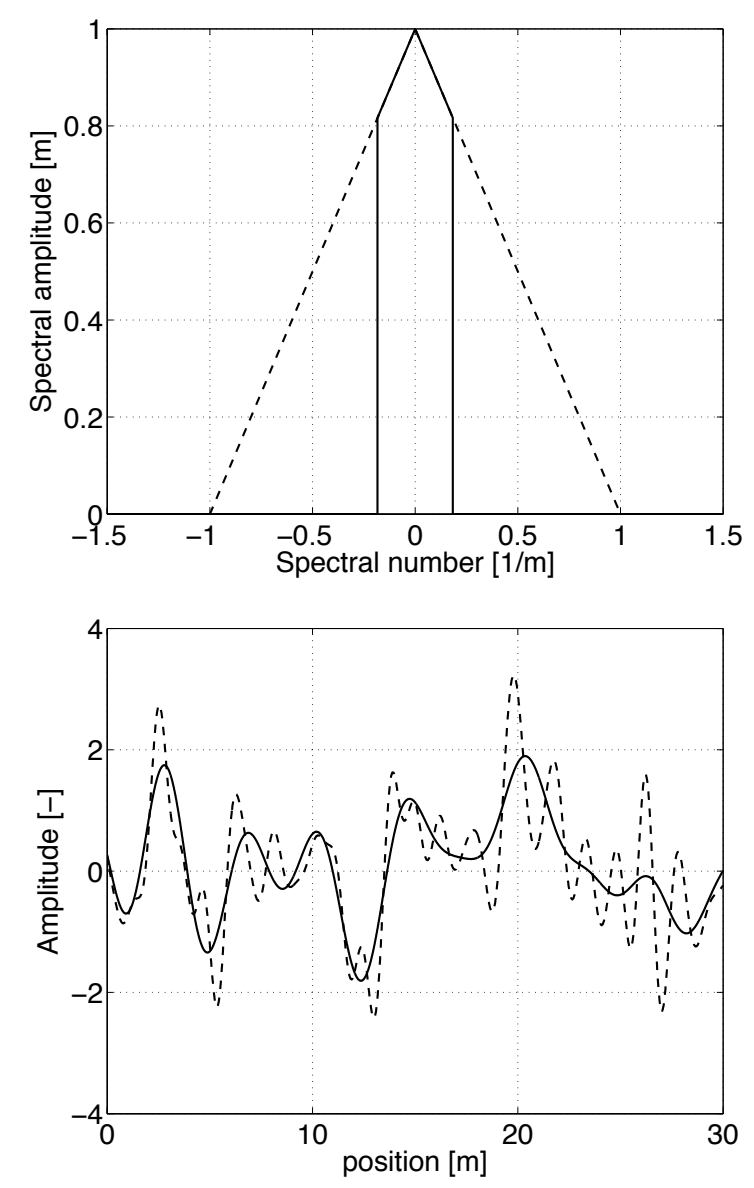

Figure 1: PSD (upper figure) and trajectories (lower figure) of two 1D centered normal fields at different scales. The dashed lines correspond to a micro-scale field $k_{m}(x)$ with triangular spectrum, unit correlation length $\ell_{m}=1 \mathrm{~m}$, and unit variance $\sigma_{m}^{2}=1(\mathrm{~N} / \mathrm{m})^{2}$. The solid lines correspond to the corresponding meso-scale random field $k_{M}(x)$ with trimmed triangular spectrum, correlation length $\ell_{M}=3 \mathrm{~m}$, and variance $\sigma_{M}^{2}=1 / 3(\mathrm{~N} / \mathrm{m})^{2}$. 

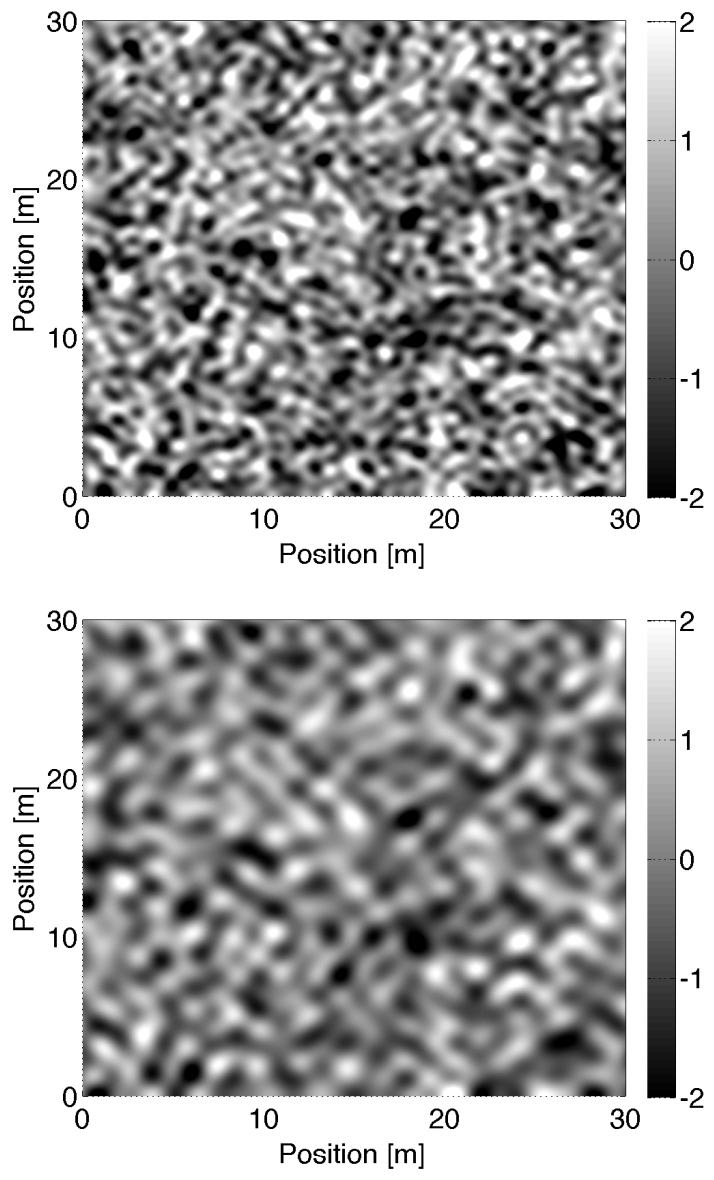

Figure 2: Trajectories of two 2D centered normal fields at different scales. The upper figure corresponds to a micro-scale field $k_{m}(x)$ with triangular spectrum, unit correlation length $\ell_{m}=1 \mathrm{~m}$, and unit variance $\sigma_{m}^{2}=1(\mathrm{~N} / \mathrm{m})^{2}$. The lower figure corresponds to the corresponding meso-scale random field $k_{M}(x)$ with trimmed triangular spectrum, correlation length $\ell_{M}=2 \mathrm{~m}$, and variance $\sigma_{M}^{2}=1 / 2(\mathrm{~N} / \mathrm{m})^{2}$.

\subsection{Meso-scale model}

From the given PSD $\hat{R}_{m}(\zeta)$ of the micro-scale parameter field $k_{m}(x)$, a hierarchy of upscaled parameter fields can be derived by trimming its tails. This trimming translates into an increase of the correlation length and a decrease of the variance. Figure 1 shows an example of such trimming on the PSD and the corresponding trajectory in 1D-model (solid lines). Figure 2 presents a similar example in 2D (lower figure). On both figures, the increase of correlation length and decrease of variance is clear. In this paper, and for a given micro-scale $\mathrm{PSD}$, we refer to meso-scale mechanical parameter (or coarse mechanical parameter) as the field $k_{M}(x)$ corresponding to the trimmed PSD, with a correlation length $\ell_{M}>\ell_{m}$, variance $\sigma_{M}^{2}<\sigma_{m}^{2}$, and average $\underline{k}_{M}$, which is a priori different from $\underline{k}_{m}$ (see the discussion in Section 2.3).

The construction above naturally introduces a segmentation of the probability space $(\Theta, \mathcal{F}, P)$ into two probability spaces $\left(\Theta_{M}, \mathcal{F}_{M}, P_{M}\right)$ and $\left(\Theta_{m}, \mathcal{F}_{m}, P_{m}\right)$ such that $k_{M}(x)$ is defined on $\mathcal{F}_{M} \otimes \mathcal{B}(\Omega)$, where $\mathcal{B}(\Omega)$ is the Borel $\sigma$-algebra generated by the open subsets of $\Omega$, $(\Theta, \mathcal{F})=\left(\Theta_{M} \times \Theta_{m}, \mathcal{F}_{M} \otimes \mathcal{F}_{m}\right)$, and $P$ is the unique product measure of $P_{m}$ and $P_{M}$, such that $\forall X_{m} \in \mathcal{F}_{m}$ and $X_{M} \in \mathcal{F}_{M}, P\left(X_{m} \times X_{M}\right)=P_{m}\left(X_{m}\right) P_{M}\left(X_{M}\right)$. We introduce the additional notations $E_{M}[\cdot]=\int_{\Theta_{M}} \cdot d P_{M}$ and $E_{m}[\cdot]=\int_{\Theta_{m}} \cdot d P_{m}$.

From this definition of the meso-scale parameter field $k_{M}(x)$, we also define a meso-scale mono-model boundary value problem, which states: find the meso-scale mono-model solution $u_{M}(x) \in \mathcal{V}_{M}$, such that

$$
a_{M}\left(u_{M}, v\right)=f(v), \forall v \in \mathcal{V}_{M},
$$

where $a_{M}: \mathcal{V}_{M} \times \mathcal{V}_{M} \rightarrow \mathbb{R}$ is defined by $a_{M}(u, v)=$ $\mathrm{E}_{M}\left[\int_{\Omega} k_{M}(x) \nabla u \cdot \nabla v d x\right]$, and $\mathcal{V}_{M}=\mathcal{L}^{2}\left(\Theta_{M}, \mathcal{H}_{0}^{1}\right)$. Endowed with appropriate inner product and norm, $\mathcal{V}_{M}$ is a Hilbert space. As before, it can be proved that the problem (2) has a unique solution $u_{M}$.

\subsection{Partial upscaling and homogenization}

Extending the upscaling process described above to the limit $\ell_{M} \rightarrow+\infty$, one obtains a deterministic homogeneous mechanical parameter $k^{*}$. In general, this parameter $k^{*}$ is not equal to the parameter obtained from classical homogenization [56, 57, 58, 59]. However, in the particular case of a micro-scale parameter with lognormal first-order marginal density in $2 \mathrm{D}$, the homogenized tensor is equal to the geometric average of the field $k^{*}=\underline{k}_{m} /\left(1+\sigma_{m}^{2} / \underline{k}_{m}^{2}\right)^{1 / 2}$. Hence, if we assume that both the micro-scale and meso-scale models follow a 
log-normal first-order marginal density, the average $\underline{k}_{M}$ of the meso-scale model can be chosen such that the two models are coherent in the sense that they have the same homogenized coefficient $k^{*}=\underline{k}_{m} /\left(1+\sigma_{m}^{2} / \underline{k}_{m}^{2}\right)^{1 / 2}=$ $\underline{k}_{M} /\left(1+\sigma_{M}^{2} / \underline{k}_{M}^{2}\right)^{1 / 2}$. With this hypothesis, a meso-scale model $k_{M}(x)$, with trimmed triangular spectrum and lognormal first-order marginal density, can be defined starting from a micro-scale model $k_{m}(x)$ for any choice of correlation length $\ell_{M}>\ell_{m}$. Using the technique described in [60], it is furthermore possible to generate realizations of the two fields $k_{M}(x)$ and $k_{m}(x)$ that correspond one to the other, realization by realization, as illustrated in Figures 1 and 2.

It should be noted that if the mechanical parameter fields $k_{m}$ and $k_{M}$ follow log-normal first-order marginal densities, they are not bounded almost surely. LaxMilgram theorem can then not be used to prove existence and uniqueness of the micro- and meso-scale mono-model solutions. Hence, we will rather use trimmed log-normal first-order marginal densities. The inferior bound $b_{\min }$ and superior bound $b_{\max }$ should be chosen far enough from unity: $0<b_{\min }<<1$ and $1>b_{\max }$, so that the distribution remains close to a log-normal one, and the analytical formula can still be used (this is only for simplicity). Also, the bounds should be chosen such that $b_{\min }=1 / b_{\max }$, so that the first-order marginal distributions of the variables and their inverse remain equal and the formula above for the 2D homogenized coefficient $k^{*}$ [61, Chap. 3] remains valid.

Note that this question of upscaling of random microscale models has been widely treated in the literature $[62,63,64,60]$. In particular, some upscaling techniques seem to provide partial upscaling naturally $[65,66]$. The choice of micro-scale and mesoscale models described in this section does not pretend to be representative of the state-of-the-art in this field. It is rather one very particular choice for which a sequence of models can be constructed analytically in a manner consistent with (classical) homogenization. Our objective in this paper does not lie in the actual process of partial upscaling and homogenization, but in the description of a coupling technique for two stochastic continuum models at two different scales.

\section{Coupling method in a stochastic framework}

This section now concentrates on this issue of coupling two stochastic continuum media. It constitutes the core of this paper. We consider again the domain $\Omega$ and now divide it into two overlapping subdomains
$\Omega_{m}$ and $\Omega_{M}$ such that $\Omega_{m} \cup \Omega_{M}=\Omega$ (see Figure 3). The Dirichlet and Neumann boundaries separate into corresponding overlapping boundaries $\Gamma_{D}^{m}, \Gamma_{D}^{M}, \Gamma_{N}^{m}$ and $\Gamma_{N}^{M}$. Note that one of the two Dirichlet boundaries may be empty. On each subdomain, a different model will be considered, with a different mechanical parameter field, namely $k_{m}(x)$ on $\Omega_{m}$ and $k_{M}(x)$ on $\Omega_{M}$. As with other similar techniques, the general idea is here to replace, where it is relevant, a fine-scale model by a coarser one in order to focus the available resources on a limited area of the domain. This section describes the coupled problem then posed on $\Omega$. This coupling problem is developed in the Arlequin framework [46, 67, 68, 69, 50, 18, 20]. This framework is based on three ingredients: (i) splitting of the domain into overlapping subdomains to which different models are attached, (ii) introduction of weight functions to dispatch the global energy among the models, (iii) imposition of a weak compatibility constraint between the solutions of the different models. The developments proposed below for a coupling between two stochastic continuum models stand mainly in the choice of the coupling operator and space (ingredient (iii) above).

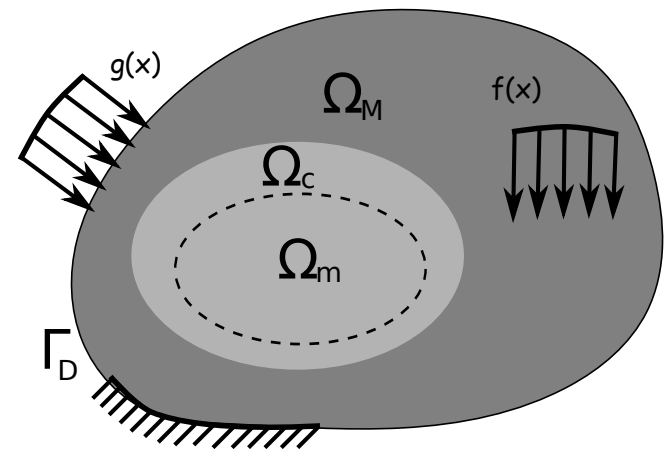

Figure 3: General setting of the Arlequin model: overlapping microscale domain $\Omega_{m}$ and meso-scale domain $\Omega_{M}$ and coupling volume $\Omega_{c}$.

\subsection{Arlequin formulation}

We choose a so-called coupling volume $\Omega_{c} \subset\left(\Omega_{m} \cap\right.$ $\Omega_{M}$ ), over which the two models are assumed to exchange information. In the particular case of homogeneous Dirichlet boundary conditions (the extension to non-homogeneous boundary conditions is obvious), the mixed Arlequin problem reads: find $\left(w_{m}, w_{M}, \Phi\right) \in$ $\mathcal{W}_{m} \times \mathcal{W}_{M} \times \mathcal{W}_{c}$ such that

$$
\begin{cases}\mathcal{A}_{m}\left(w_{m}, v\right)+C(\Phi, v)=f_{m}(v), & \forall v \in \mathcal{W}_{m} \\ \mathcal{A}_{M}\left(w_{M}, v\right)-C(\Phi, v)=f_{M}(v), & \forall v \in \mathcal{W}_{M}, \\ C\left(\Psi, w_{m}-w_{M}\right)=0, & \forall \Psi \in \mathcal{W}_{c}\end{cases}
$$


where the bilinear forms $\mathcal{A}_{m}: \mathcal{W}_{m} \times \mathcal{W}_{m} \rightarrow \mathbb{R}, \mathcal{A}_{M}$ : $\mathcal{W}_{M} \times \mathcal{W}_{M} \rightarrow \mathbb{R}$, and $C: \mathcal{W}_{c} \times \mathcal{W}_{c} \rightarrow \mathbb{R}$ are defined by $\mathcal{A}_{m}(w, v)=\mathrm{E}\left[\int_{\Omega_{m}} \alpha_{m} k_{m}(x) \nabla w \cdot \nabla v d x\right], \mathcal{A}_{M}(w, v)=$ $\mathrm{E}_{M}\left[\int_{\Omega_{M}} \alpha_{M} k_{M}(x) \nabla w \cdot \nabla v d x\right]$, and

$$
C(w, v)=\mathrm{E}\left[\int_{\Omega_{c}}(w v+\kappa \nabla w \cdot \nabla v) d x\right],
$$

where $\kappa$ is a constant essentially introduced for dimensionality purposes [68], and where the linear forms $f_{m}: \mathcal{W}_{m} \rightarrow \mathbb{R}$ and $f_{M}: \mathcal{W}_{M} \rightarrow \mathbb{R}$ are defined, respectively, by $f_{m}(v)=\int_{\Omega_{m}} \alpha_{m} f \mathrm{E}[v] d x+\int_{\Gamma_{D}^{m}} \alpha_{m} g \mathrm{E}[v] d x$ and $f_{M}(v)=\int_{\Omega_{M}} \alpha_{M} f \mathrm{E}[v] d x+\int_{\Gamma_{D}^{M}} \alpha_{M} g \mathrm{E}[v] d x$. The weights are chosen such that:

$$
\begin{cases}\alpha_{m}, \alpha_{M} \geq 0 & \text { in } \Omega_{m} \cup \Omega_{M}, \\ \alpha_{m}+\alpha_{M}=1 & \text { in } \Omega_{m} \cup \Omega_{M}, \\ \alpha_{m}, \alpha_{M} \text { constant } & \text { in }\left(\Omega_{m} \cup \Omega_{M}\right) \backslash \Omega_{c} .\end{cases}
$$

The functional spaces are $\mathcal{W}_{M}=\mathcal{L}^{2}\left(\Theta_{M}, \mathcal{H}_{M}^{1}\right)$ with $\mathcal{H}_{M}^{1}=\left\{w \in \mathcal{H}^{1}\left(\Omega_{M}\right), w_{\mid \Gamma_{D}^{M}}=0\right\}$, for the meso-scale space, and $\mathcal{W}_{m}=\mathcal{L}^{2}\left(\Theta, \mathcal{H}_{m}^{1}\right)$, with $\mathcal{H}_{m}^{1}= \begin{cases}w \in\end{cases}$ $\left.\mathcal{H}^{1}\left(\Omega_{m}\right), w_{\mid \Gamma_{D}^{m}}=0\right\}$, for the micro-scale space. Note that the equalities on the boundary conditions should be understood as $P_{M}$-almost surely and almost-everywhere in $\Omega_{M}$, and $P$-almost surely and almost-everywhere in $\Omega_{m}$, respectively. The so-called mediator space $\mathcal{W}_{c}$ is defined in the Section 3.2.

One can consider that the system (3) consists of three equations: (i) one governing the behavior of the microscale model, weighted by $\alpha_{m}(x)$ and with a loading arising in the coupling volume $\Omega_{c}$ embodied in the operator $C$, (ii) one governing the behavior of the meso-scale model, weighted by $\alpha_{M}(x)$ and with a loading opposite to the previous in the coupling volume $\Omega_{c}$, and (iii) one enforcing the weak compatibility between the two solutions $w_{m}(x)$ and $w_{M}(x)$.

\subsection{Mediator space}

There are two natural choices for the mediator space: (i) the restriction of the functional space for the micro-scale model over the coupling volume $W_{c}^{(i)}=$ $\mathcal{L}^{2}\left(\Theta_{M}, \mathcal{H}^{1}\left(\Omega_{c}\right)\right)$, and (ii) the restriction of the functional space for the meso-scale model over the coupling volume $\mathcal{W}_{c}^{(i i)}=\mathcal{L}^{2}\left(\Theta, \mathcal{H}^{1}\left(\Omega_{c}\right)\right)$.

However, the former choice (i) leads to an unstable mixed problem when no Dirichlet boundary condition is enforced on the micro-scale model $\left(\Gamma_{D}^{m}=\emptyset\right)$ for example. This is an important case, that includes in particular zooms $\Omega_{m} \subset \Omega_{M}$ with $\partial \Omega_{m} \cap \partial \Omega=\emptyset$.
The latter choice of mediator space (ii) does lead to a stable mixed formulation. The coupling operator $C$ induces in that case solutions that are such that $w_{m}=w_{M}$ almost everywhere in $\Omega_{c}$ and $P$-almost surely. Based on observations on Figure 4 for example, we consider that this is too demanding on the solutions. Figure 4 presents an example of micro-scale mono-model and meso-scale mono-model solutions obtained by solving Eq. (1) and (2), respectively, for a realization of $k_{m}$ and a corresponding upscaled version of it $k_{M}$ for a classical $1 \mathrm{D}$ traction problem. The parameter fields correspond one to other, although $k_{m}$ presents a higher frequency content than $k_{M}$, and we observe that the solutions behave similarly. Choosing $\mathcal{W}_{c}^{(i i)}$ as mediator space would essentially impose that the two solutions overlap in the coupling volume, among other things limiting the frequency content of the micro-scale solution $w_{m}$ to that of the coarser meso-scale solution. An appropriate choice of mediator space should rather try to enforce a weaker equality between $u_{m}$ and $u_{M}$ in the coupling volume, allowing the two solutions $w_{m}$ and $w_{M}$ of the coupled problem (3) to oscillate at different frequencies.

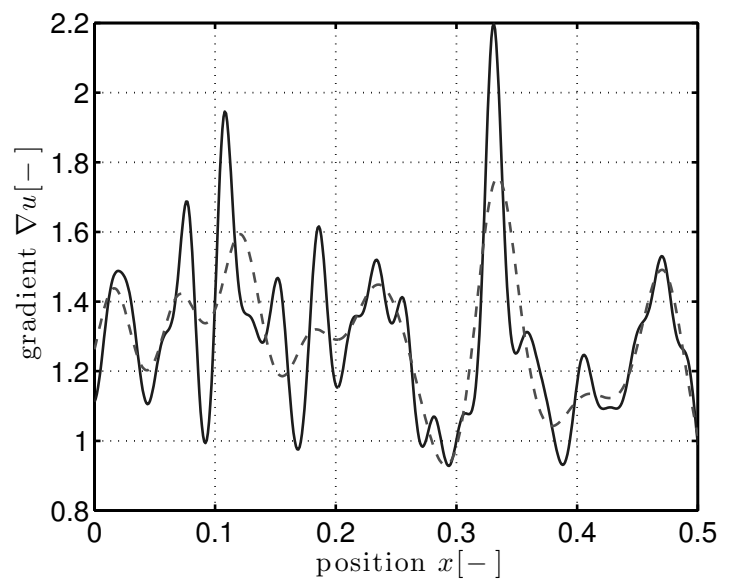

Figure 4: Gradients of the micro-scale mono-model solution $u_{m}(x)$ (solid line) and meso-scale mono-model solution $u_{M}(x)$ (dashed line) for a bar under traction and unit bulk load. The micro-scale mechanical parameter follows a (trimmed) log-normal first-order marginal density, triangular PSD with $\ell_{m}=3 \times 10^{-3} \mathrm{~m}$ and $\sigma_{m}^{2}=2.5 \times$ $10^{-2}(\mathrm{~N} / \mathrm{m})^{2}$. The meso-scale mechanical parameter is obtained as described in Section 2 for $\ell_{M}=4.5 \times 10^{-3} \mathrm{~m}$ and $\sigma_{M}^{2}=1.65 \times$ $10^{-2}(\mathrm{~N} / \mathrm{m})^{2}$.

The mediator space is therefore chosen as the sum of a stochastic field related to the meso-scale variation, which imposes a strong equality constraint on the lowfrequency parts of $u_{m}$ and $u_{M}$, and a stochastic variable related to the high-frequency fluctuations, impos- 
ing only a weak constraint on that part of the spectrum of $u_{m}$ and $u_{M}$ :

$$
\mathcal{W}_{c}=\mathcal{L}^{2}\left(\Theta_{M}, \mathcal{H}^{1}\left(\Omega_{c}\right)\right)^{*} \oplus \mathcal{L}^{2}\left(\Theta_{m}, \mathbb{R}\right),
$$

where the Bochner space $\mathcal{L}^{2}\left(\Theta_{M}, \mathcal{H}^{1}\left(\Omega_{c}\right)\right)^{*}=$ $\mathcal{L}^{2}\left(\Theta_{M}, \mathcal{H}^{1}\left(\Omega_{c}\right)\right) \backslash\left\{v \quad \mid \mathrm{E}_{M}\left[\int_{\Omega_{c}} v d x\right]=0\right\}$ explicitly excludes function with a deterministic spatial average. This exclusion is necessary in order that there be no intersection between the two spaces above. This also explains the introduction of matrices $S_{c}$ for the numerical approximation of system (3) (see Section 4 for more details, and in particular Equation (11)).

After some algebraic manipulations, this choice of the coupling space translates into the following weak equality constraint in the last line of Equation (3): $\forall \Psi=$ $\psi\left(x, \theta_{M}\right)+\theta_{m} \in \mathcal{W}_{c}$, with $\psi\left(x, \theta_{M}\right) \in \mathcal{L}^{2}\left(\Theta_{M}, \mathcal{H}^{1}\left(\Omega_{c}\right)\right)^{*}$, $\theta_{m} \in \mathcal{L}^{2}\left(\Theta_{m}, \mathbb{R}\right)$,

$$
\begin{aligned}
0=C\left(\mathrm{E}_{m}[\Psi], w_{M}-\mathrm{E}_{m}\left[w_{m}\right]\right) & \\
& -\mathrm{E}_{m}\left[\theta_{m} \int_{\Omega_{c}} \mathrm{E}_{M}\left[w_{m}-\mathrm{E}_{m}\left[w_{m}\right]\right] d x\right]
\end{aligned}
$$

On the one hand, this equation imposes that the solutions $w_{m}$ and $w_{M}$ be equal almost everywhere in $\Omega_{c}$ and almost surely with respect to the low-frequency fluctuations of the medium $\left(P_{M}\right.$-almost surely). On the other hand, it imposes that the average in space (over $\Omega_{c}$ ) of the high frequency fluctuations of $w_{m}$ vanishes $P_{m^{-}}$ almost surely.

\subsection{Arlequin coupled solution}

Each of the solutions $w_{m}$ and $w_{M}$ is spatially supported only over a subdomain of $\Omega$. We discuss here how to transform these localized solutions into global solutions. The micro-scale information is available only within $\Omega_{m}^{f}=\Omega_{m} \backslash \Omega_{c}$, where the micro-scale parameter $k_{m}$ is actually defined, and where the weight function actually puts emphasis on it. The micro-scale coupled solution is therefore only defined within this subdomain $u_{m}^{\text {arl }} \in \mathcal{L}^{2}\left(\Theta, \mathcal{H}_{0}^{1}\left(\Omega_{m}^{f}\right)\right)$ as

$$
u_{m}^{\mathrm{arl}}=\alpha_{m} w_{m}+\alpha_{M} w_{M},
$$

assuming extension by 0 of the functions wherever they are not naturally defined. On the other hand, the meso-scale information is available everywhere, either through $k_{M}$, or through $k_{m}$ with also contains that mesoscale information. We can therefore define a global meso-scale coupled solution $u_{M}^{\text {arl }} \in \mathcal{L}^{2}\left(\Theta_{M}, \mathcal{H}_{\text {brok }}^{1}\right)$ as

$$
u_{M}^{\mathrm{arl}}=\alpha_{m} \mathrm{E}_{m}\left[w_{m}\right]+\alpha_{M} w_{M},
$$

where $\mathcal{H}_{\text {brok }}^{1}=\left\{w \in \mathcal{H}_{\text {brok }}^{1}(\Omega), w_{\mid \Gamma_{D}}=0\right\}$, and discontinuities in the solution $u_{M}^{\text {arl }}$ may appear when the weight functions $\alpha_{m}(x)$ and $\alpha_{M}(x)$ are not continuous.

\section{Numerical implementation}

To approximate the solution $\left(w_{m}, w_{M}, \Phi\right)$ of Equation (3), a mesh $\mathcal{T}_{m}$ of $n_{m}$ elements $\mathcal{E}_{m}$ is constructed over $\Omega_{m}$, a mesh $\mathcal{T}_{M}$ of $n_{M}$ elements $\mathcal{E}_{M}$ is constructed over $\Omega_{M}$, and a mesh $\mathcal{T}_{c}$ of $n_{c}$ elements $\mathcal{E}_{c}$ is constructed over $\Omega_{c}$. The following functional spaces are introduced: $\mathcal{V}_{m}^{H}=\left\{v \in \mathbb{P}^{1}\left(\mathcal{E}_{m}\right), v_{\mid \Gamma_{D}^{m}}=0\right\}, \mathcal{V}_{M}^{H}=\{v \in$ $\left.\mathbb{P}^{1}\left(\mathcal{E}_{M}\right), v_{\mid \Gamma_{D}^{M}}=0\right\}$ and $\mathcal{V}_{c}^{H}=\left\{v \in \mathbb{P}^{1}\left(\mathcal{E}_{c}\right)\right\}$, composed of linear polynomials on the elements of the different meshes (higher-order polynomials could obviously be used). We associate to these functional spaces the bases: $\left\{v_{i}^{m}\right\}_{1 \leq i \leq n_{m}},\left\{v_{i}^{M}\right\}_{1 \leq i \leq n_{M}}$, and $\left\{v_{i}^{c}\right\}_{1 \leq i \leq n_{c}}$.

The mixed system (3) is transformed, after space discretization, into the random matrix system:

$$
\mathrm{E}\left[\mathrm{A}\left(\theta_{m}, \theta_{M}\right) \mathrm{U}\left(\theta_{m}, \theta_{M}\right)\right]=\mathrm{F},
$$

where $\theta_{m}$ and $\theta_{M}$ indicate dependency on $\Theta_{m}$ and $\Theta_{M}$, respectively, and where

$$
\begin{aligned}
& \mathrm{A}\left(\theta_{m}, \theta_{M}\right)= \\
& \left(\begin{array}{ccccc}
A_{m}\left(\theta_{m}, \theta_{M}\right) & 0 & C_{m} & S_{m} & 0 \\
0 & A_{M}\left(\theta_{M}\right) & -C_{M} & -S_{M} & 0 \\
C_{m}^{T} & -C_{M}^{T} & 0 & 0 & S_{c}^{T} \\
S_{m}^{T} & -S_{M}^{T} & 0 & 0 & 0 \\
0 & 0 & S_{c} & 0 & 0
\end{array}\right),
\end{aligned}
$$

with, for $n=\{m, M\}$,

$$
\begin{gathered}
A_{n, i j}(\theta)=\int_{\Omega_{n}} \alpha_{n}(x) k_{n}(x, \theta) \nabla v_{i}^{n}(x) \cdot \nabla v_{j}^{n}(x) d x, \\
C_{n, i j}=\int_{\Omega_{c}} v_{i}^{c} v_{j}^{n}+\kappa \nabla v_{i}^{c} \cdot \nabla v_{j}^{n} d x,
\end{gathered}
$$

and, for $n=\{m, M, c\}$,

$$
S_{n, j}=\int_{\Omega_{c}} v_{j}^{n} .
$$

The load vector is $\mathrm{F}=\left[\begin{array}{lllll}\mathrm{F}_{m} & \mathrm{~F}_{M} & 0 & 0 & 0\end{array}\right]^{T}$, where, for $n=\{m, M\}, \mathrm{F}_{n, j}=\int_{\Omega_{n}} \alpha_{n} f v_{j}^{n} d x+\int_{\Gamma_{D}^{n}} \alpha_{n} g v_{j}^{n} d x$. The unknown vector is defined by $\mathrm{U}\left(\theta_{m}, \theta_{M}\right)=$ $\left[\begin{array}{lllll}U_{m}\left(\theta_{m}, \theta_{M}\right) & U_{M}\left(\theta_{M}\right) & \Psi_{M}\left(\theta_{M}\right) & \Psi_{m}\left(\theta_{m}\right) & \Lambda\end{array}\right]^{T}$, where the unknown fields are such that $w_{m}\left(x, \theta_{m}, \theta_{M}\right)=$ $U_{m, j}\left(\theta_{m}, \theta_{M}\right) v_{j}^{m}(x), \quad w_{M}\left(x, \theta_{M}\right)=U_{M, j}\left(\theta_{M}\right) v_{j}^{M}(x)$, $\Phi\left(x, \theta_{m}, \theta_{M}\right)=\Psi_{M, j}\left(\theta_{M}\right) v_{j}^{c}(x)+\Psi_{m}\left(\theta_{m}\right)$, and the additional scalar $\Lambda$ is a Lagrange multiplier ensuring that $\int_{\Omega_{c}} \Psi_{M, j} v_{j}^{c} d x=0$. This last equation is necessary to restrict the elements of $\mathcal{L}^{2}\left(\Theta_{M}, \mathcal{H}^{1}\left(\Omega_{c}\right)\right)^{*}$ to non-constant functions, as described in Section 3.2.

This matrix system can be solved in two different ways. This first consists in solving it using a looped 
Monte Carlo approach. For each realization of $k_{M}$, a number of Monte Carlo realizations of $k_{m}$ are generated corresponding to that $k_{M}$ and the statistics (with respect to $\Theta_{m}$ ) of the solution are estimated for a fixed $\theta_{M}$ using a condensation approach similar to what is done in [18]. Solving for several realizations of $k_{M}$ then provides the statistics with respect to both $\Theta_{m} \times \Theta_{M}$. In that approach, the matrices that are inverted for each of the Monte Carlo samples are small, since the micro-scale domain is expected to be much smaller than the mesoscale domain. However, these matrices are Schur complements and in general are not sparse, so that iterative inversion algorithms are usually less efficient.

Another approach consists in considering that each entry of the solution vector $U$ is a random variable in $\mathcal{L}^{2}(\Theta, \mathbb{R})$, solving the full system for each Monte Carlo sample, and obtain the solutions by taking the appropriate averages for each element of $U$. The inconvenient of that approach is that the matrices to invert are larger, but on the other hand, they are sparse. From first tests that we have performed, it seems that the second approach is more interesting in terms of CPU time. However, these tests were performed for rather large $\Omega_{m}$ (with respect to $\Omega_{M}$ ) so that they may not be fully objective. More tests are required to clarify this issue.

\section{Numerical examples}

We present here two applications. The first one consists in a simple 1D traction problem. The 1D setting allows to compare the micro-scale mono-model, mesoscale mono-model and coupled solutions. A second application presents a $2 \mathrm{D}$ problem, more relevant for real problems, where localization of stresses calls for a micro-scale model localized around a geometrical feature.

\section{1. $1 D$ bar in traction}

The first application is mainly introduced to compare the solutions obtained by solving the micro-scale mono-model boundary value problem (1), the mesoscale mono-model boundary value problem (2) and the Arlequin mixed problem (3). In particular, the 1D setting allows to observe in detail the behavior of the different solutions in the coupling volume. We consider a bar defined over the domain $\Omega=\left[\begin{array}{ll}0 & 1\end{array}\right]$ and submitted to a constant unit bulk load $f=1$ and to a differential of Dirichlet boundary conditions. All quantities are non-dimensionalized for notational simplicity.
Micro-scale mono-model problem. We consider a micro-scale mechanical parameter $k_{m}(x)$ statistically homogeneous, with (trimmed, see Section 2.3) lognormal first order marginal law, expectation $\underline{k}_{m}=1$, variance $\sigma_{m}^{2}=2.5 \times 10^{-2}$, triangular PSD and correlation length $\ell_{m}=1 \times 10^{-2}$. The micro-scale mono-model solution $u_{m}$ verifies, $P$-almost surely, and almost everywhere in $\Omega$ :

$$
\frac{d}{d x}\left(k_{m} \frac{d u_{m}}{d x}\right)+f=0,
$$

and the boundary conditions are $u_{m}(0)=0$ and $u_{m}(1)=$ 1 almost surely. The analytical solution for the microscale mono-model solution is:

$$
u_{m}(x)=\int_{0}^{x} \frac{k^{*}-f x^{\prime}}{k_{m}\left(x^{\prime}\right)} d x^{\prime},
$$

where $k^{*}$ verifies $1=\int_{0}^{1}\left(k^{*}-f x^{\prime}\right) / k\left(x^{\prime}\right) d x^{\prime}$ almost surely, and can be approximated when $\ell_{m}<|\Omega|$ by the homogenized parameter, which is equal to the harmonic average in 1D: $k^{*}=\mathrm{E}\left[k_{m}^{-1}\right]^{-1}=\underline{k}_{m} /\left(1+\sigma_{m}^{2} / \underline{k}_{m}^{2}\right) \approx$ 0.976 . Finally, we have the following equation for the gradient:

$$
\frac{d u_{m}}{d x}=\frac{k^{*}-x}{k_{m}(x)}
$$

Note that we have $\mathrm{E}\left[u_{m}\right]=x(3-x) /\left(2 k^{*}\right)$ and $\mathrm{E}\left[d u_{m} / d x\right]=(3-2 x) /\left(2 k^{*}\right)$.

Using a linear finite element method with 1000 homogeneous interval elements, and 10,000 Monte Carlo samples, one can estimate the statistics of the microscale mono-model solution. Figure 5 displays the average, the $90 \%$-confidence interval, and one realization for that solution and its gradient. A $p c$-confidence interval for a random variable $X$ is defined as:

$$
\mathrm{IC}_{X}=\mathrm{E}[X] \pm \sqrt{\frac{\mathrm{E}\left[(X-\mathrm{E}[X])^{2}\right]}{1-p c}}
$$

On Figure 5, one can observe the rapid fluctuations of the micro-scale mono-model solution. As the observations are much clearer for the gradient than for the solution itself, we will only observe gradients from now on.

Meso-scale mono-model problem. We now consider a meso-scale model for the same 1D traction bar problem. We consider a meso-scale parameter field $k_{M}(x)$, obtained by upscaling, as described in Section 2, of the micro-scale parameter field $k_{m}(x)$ described above. The meso-scale parameter field follows a (trimmed) lognormal first-order marginal law with a trimmed triangle 

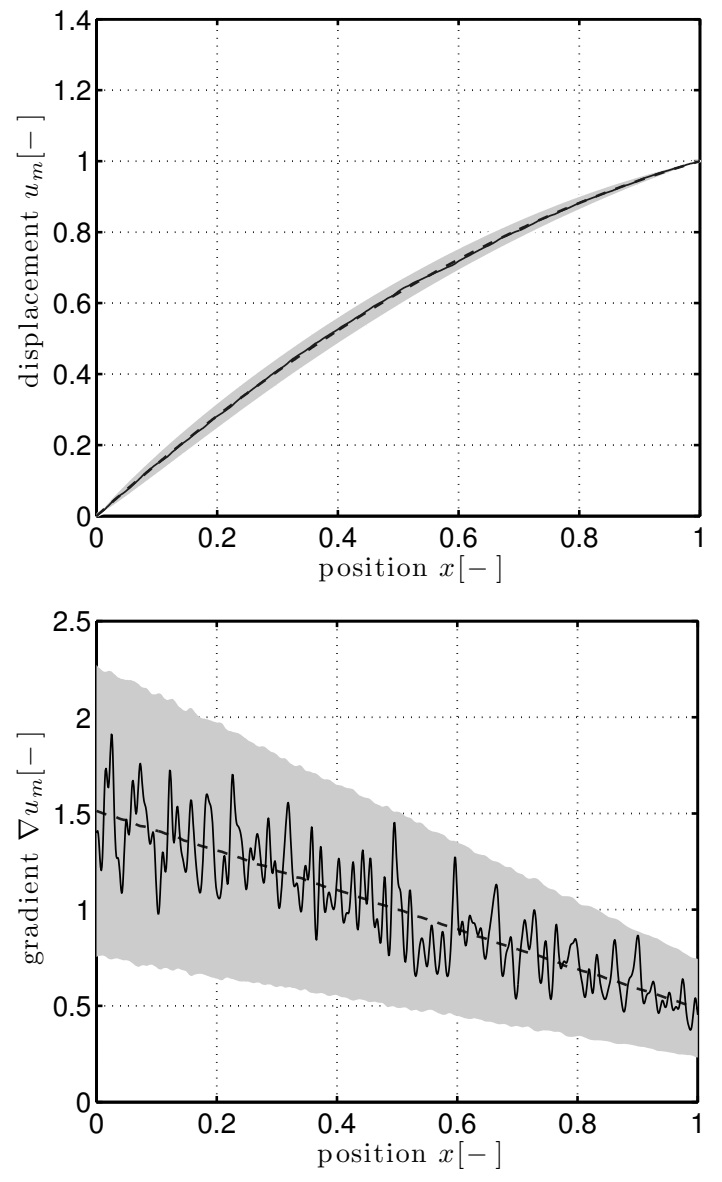

Figure 5: Micro-scale mono-model solution (upper figure) and its gradient (lower figure): average (dashed lines), 90\%-confidence interval (grey shades) and one realization (solid lines).
PSD with correlation length set to $\ell_{M}=2 \times 10^{-1}$, variance $\sigma_{M}^{2}=1.2 \times 10^{-3}$ and average value $\underline{k}_{M}=0.976$ (in order to have the same homogenized coefficient $k^{*}$ for both models). The meso-scale mono-model boundary value problem is the same as in Equation (15), using the meso-scale parameter field $k_{M}(x)$, and with a solution $u_{M}$. The statistics of that meso-scale mono-model solution are estimated using the same numerical method and parameters as for the micro-scale mono-model. Figure 6 displays the average, the $90 \%$-confidence interval, and one realization for the gradient of that solution. As expected, the fluctuations of the gradient of the mesoscale mono-model solution $u_{M}$ are smoother than for the micro-scale mono-model solution $u_{m}$, and the confidence interval is smaller.

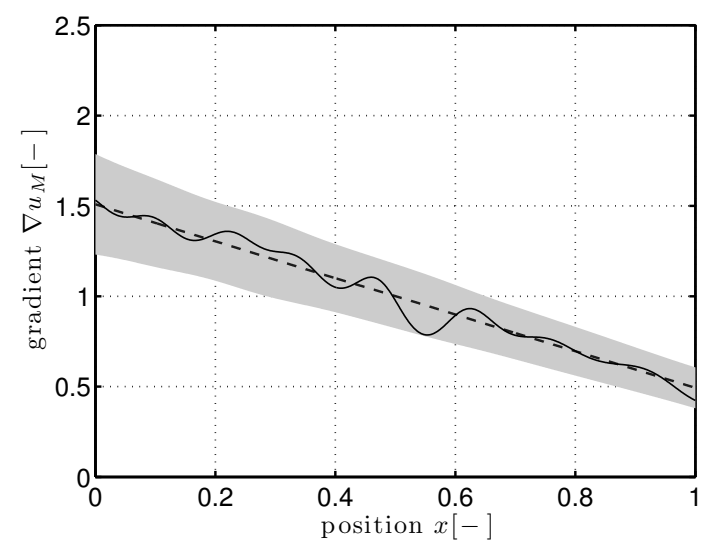

Figure 6: Gradient of the meso-scale mono-model solution $u_{M}(x)$ : average (dashed line), 90\%-confidence interval (grey shade) and one realization (solid line).

Arlequin coupled problem. We finally turn to an Arlequin coupled model of the same $1 \mathrm{D}$ traction bar problem. The meso-scale subdomain is set to $\Omega_{M}=[0.1,1]$, the micro-scale subdomain to $\Omega_{m}=[0,0.2]$, and the coupling domain to $\Omega_{c}=[0.1,0.2]$. We consider the same parameter fields $k_{m}(x)$ and $k_{M}(x)$ as for the monomodel cases. To accommodate the oscillations of each model, $\Omega_{M}$ is discretized into 45 elements of length 0.02 , and $\Omega_{m}$ is discretized into 200 elements of length $10^{-3}$. For simplicity, the two meshes are embedded in $\Omega_{c}$, although this is not a restriction of the method. The mesh corresponding to the spatial discretization of the mediator space $\mathcal{W}_{c}$ follows the mesh of $\Omega_{M}$ (therefore with 5 elements). The number of Monte Carlo samples is set to 100,000 , using 1000 samples of $k_{M}(x)$ and, for each sample of $k_{M}(x), 100$ samples of $k_{m}(x)$, as described in Section 4. The parameter $\kappa$ is set to $10^{-3}$. 
The weight functions are set as in Equation (5), and linear between $10^{-3}$ and $\left(1-10^{-3}\right)$ in the coupling zone. The total computation time was $200 \mathrm{~s}$ on a four $3.3 \mathrm{GHz}$ processor computer.

The raw output of the Arlequin coupled system are the two solutions $w_{m}(x)$ and $w_{M}(x)$. Their gradients are plotted in Figure 7 without any post-treatment. For one realization, the expected behavior that $w_{M}$ be a lowfrequency filtered version of $w_{m}$ is observed. Also, as induced by Equation (7), the ensemble average (with respect to $P$ ) of the two solutions matches in the coupling domain $\Omega_{c}$.

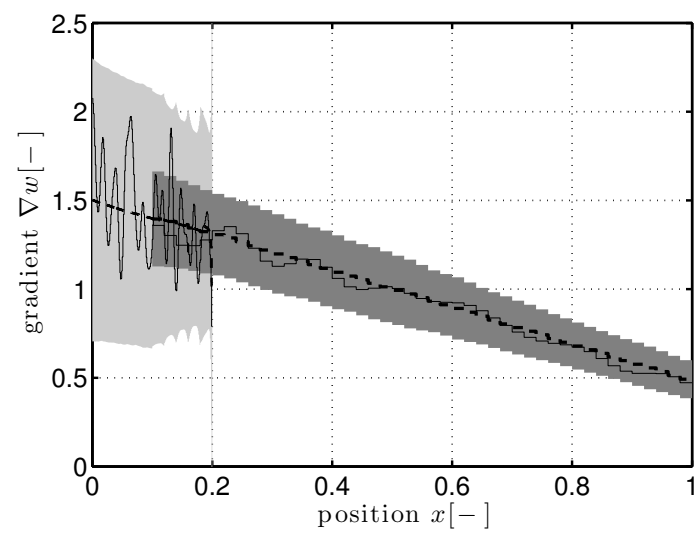

Figure 7: Gradients of the micro-scale $w_{m}(x)$ and meso-scale $w_{M}(x)$ solutions of the Arlequin coupled problem: average (dashed lines), $90 \%$-confidence interval (grey shades) and one realization (solid lines).

Comparisons and comments. The micro-scale and meso-scale coupled solution can be constructed according to Equations (8) and (9), respectively. As stated in its definition, the micro-scale coupled solution $u_{m}^{\mathrm{arl}}$ is only defined in $\Omega_{m} \backslash \Omega_{c}$, where the micro-scale information is actually available and put emphasis on (through the weight functions). On the contrary, the meso-scale coupled solution $u_{M}^{\text {arl }}$ is reconstructed over the entire domain. The two coupled solutions $u_{m}^{\text {arl }}$ and $u_{m}^{\text {arl }}$ are plotted in Figures 8 and 9, respectively. On the same figures, each of them is compared to its mono-model counterpart, in terms of average and confidence interval. A remarkable match is observed. It is interesting to note in Figure 9 that there is a smooth transition in the coupling area $\Omega_{c}$ between a gradient defined over large elements (with gradient constant over length 0.2 ) and gradient defined over small elements (with gradient constant over length 0.001 ). This smooth transition is due to the introduction of the weight functions that balance the energy between two meshes with different sizes.

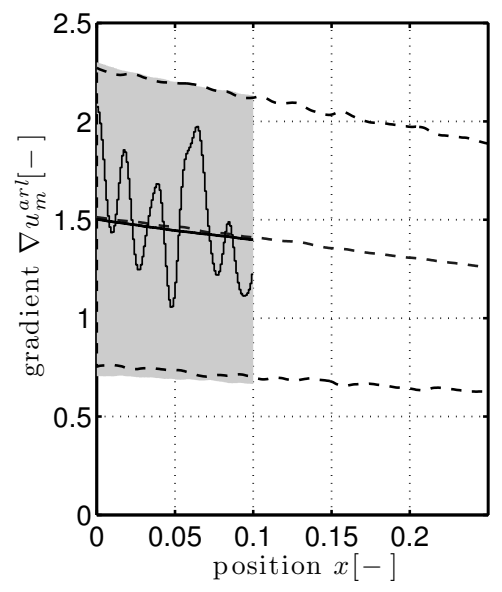

Figure 8: Comparison of the gradient of the micro-scale mono-model solution $u_{m}(x)$ and of the micro-scale coupled solution $u_{m}^{\text {arl }}$ : average (dashed and solid lines, respectively), 90\%-confidence interval (dashed line and grey shade, respectively) and one realization of the Arlequin solution (solid line).

\section{2. $2 D$ indented plate}

Let now consider the example of a plate in traction. The plate occupies a domain $\Omega$ that is inside the box $[-1,1] \times[0,1]$. This plate is indented by a notch of 1 unit length width and 0.5 unit length depth. There is no bulk modulus. The boundary conditions are $u(x=-1, y)=0$ and $u(x=1, y)=2$, and $\nabla u \cdot n=0$ on the other edges ( $n$ being the normal to the edge). The aim is to localize the full stochastic model in the bottom of the notch, the meso-scale model expresses itself on the remaining of the structure. The mechanical parameter is chosen to follow a lognormal law of unit mean and variance $\sigma_{m}^{2}=0.025$ with a triangular correlation model of correlation length $\ell_{m}=0.05$. The micro-scale area is localized to $\Omega_{m}=\ldots$, nombre d elment The meso-scale model expresses itself on the remaining of the domain. Its correlation length is $\ell_{M}=0.1$ and its variance $\sigma_{M}^{2}=0.01$ . The domain is meshed with triangular element with maximum edge size equal to 0.1 . The mesh is refined near the micro-scale zone in order to have an efficient coupling. The quantities of interest will be the displacement and its gradient (proportional to the stress). Results will be displayed on the entire structure and along the line $y=0.5$. 


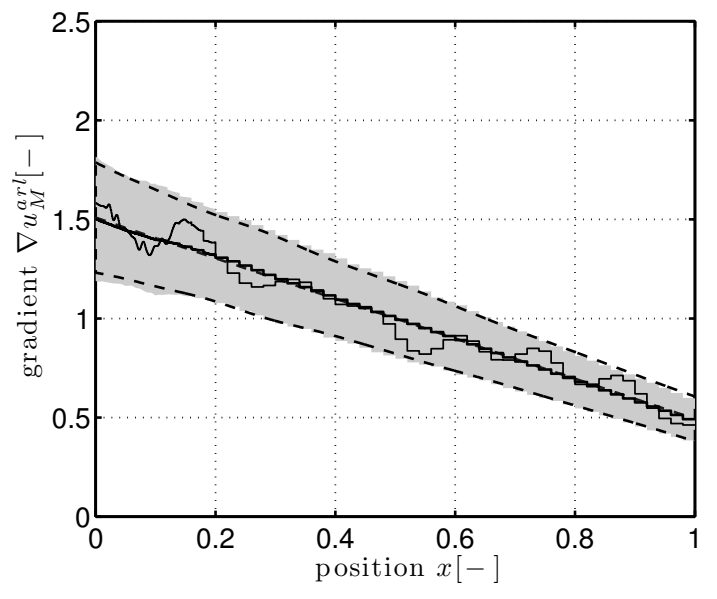

Figure 9: Comparison of the gradient of the meso-scale mono-model solution $u_{M}(x)$ and of the meso-scale coupled solution $u_{M}^{\mathrm{arl}}$ : average (dashed and solid lines, respectively), 90\%-confidence interval (dashed line and grey shade, respectively) and one realization of the Arlequin solution (solid line).

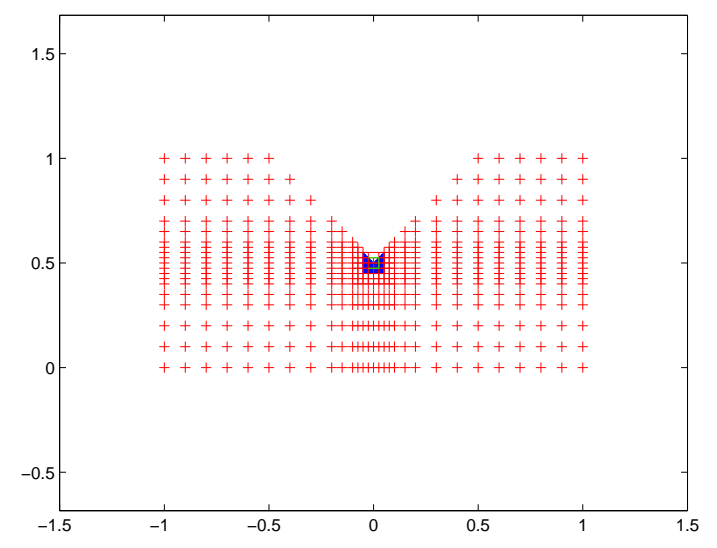

Figure 10: Coarse (red points) : $\Delta x=\Delta y=0.1, \ell_{1}=0.5, \gamma=0.2$, fine mesh (blue lines): $\Delta x=\Delta y=0.01, \ell_{2}=0.05$, coupling area between green and red lines.

\section{Conclusion}

We presented here a novel coupling approach for the approximation solution of complex multiscale stochastic problems. This coupling method is based on the Arlequin framework, with a particular choice of mediator space that can be seen as an extension of a stochasticdeterministic coupling space introduced in $[50,18]$. The chosen coupling strategy ensures that the solutions of the micro- and meso-scale models are equal almost everywhere in the coupling volume and almost surely with respect to the low-frequency fluctuations of the medium, and that the average in space of the high frequency fluctuations of the micro-scale solution vanishes. The numerical implementation is performed using two Monte-Carlo loops intertwined.

As this coupling approach stands in the Arlequin framework, it can be adapted to the case when the two models are different, in the sense that they are governed by different sets of partial differential equations. For example, it may be interesting to consider the coupling of a micro-scale 3D elastic model with a meso-scale beam model. Likewise, atomistic-to-continuum coupling approach may be approached. Although the mediator spaces should be modified accordingly, this type of coupled problems, with appropriate levels of randomness on each subdomain, would be extremely interesting in terms of applications.

\section{Acknowledgements}

This work was partially supported by the ANR project TYCHE (Advanced methods using stochastic modeling in high dimension for uncertainty modeling, quantification and propagation in computational mechanics of solids and fluids), with project number ANR2010-BLAN-0904.

\section{References}

[1] Matthies HG, Keese A. Galerkin methods for linear and nonlinear elliptic stochastic partial differential equations. Comp Meths Appl Mech Engr 2005;194(12-16):1295-331. doi: 10.1016/j.cma.2004.05.027.

[2] Frauenfelder P, Schwab C, Todor RA. Finite elements for elliptic problems with stochastic coefficients. Comp Meths Appl Mech Engr 2005;194(2-5):205-28. doi: 10.1016/j.cma.2004.04.008.

[3] Nouy A. Recent developments in spectral stochastic methods for the numerical solution of stochastic partial differential equations. Arch Comp Meths Engr 2009;16(3):251-85. doi: 10.1007/s11831-009-9034-5.

[4] Xiu D. Fast numerical methods for stochastic computations: a review. Comm Comp Phys 2009;5(2-4):242-72. 
[5] Le Maître OP, Knio OM. Spectral methods for uncertainty quantification: with applications to computational fluid dynamics. Springer; 2010

[6] Babuška I, Nobile F, Tempone R. A stochastic collocation method for elliptic partial differential equations with random input data. SIAM Rev 2010;52(2):317-55. doi: $10.1137 / 100786356$.

[7] Ghanem RG, Kruger R. Numerical solution of spectral stochastic finite element systems. Comp Meths Appl Mech Engr 1996;129(3):289-303. doi:10.1016/0045-7825(95)00909-4.

[8] Pellissetti MF, Ghanem RG. Iterative solution of systems of linear equations arising in the context of stochastic finite elements. Adv Engng Sftw 2000;31(8-9):607-16. doi:10.1016/S09659978(00)00034-X.

[9] Desceliers C, Ghanem RG, Soize C. Polynomial chaos representation of a stochastic preconditioner. Int J Numer Meths Engr 2005;64(5):618-34. doi:10.1002/nme.1382.

[10] Ullmann E. A Kronecker product preconditioner for stochastic Galerkin finite element discretizations. SIAM J Sci Comp 2010;32(2):923-46. doi:10.1137/080742853.

[11] Wheeler MF, Wildey T, Yotov I. A multiscale preconditioner for stochastic mortar mixed finite elements. Comp Meths Appl Mech Engr 2011;200(9-12):1251-62. doi: 10.1016/j.cma.2010.10.015

[12] Doostan A, Ghanem RG, Red-Horse J. Stochastic model reduction for chaos representation. Comp Meths Appl Mech Engr 2007;196(37-40):3951-66. doi:10.1016/j.cma.2006.10.047.

[13] Arnst M, Ghanem R. Probabilistic equivalence and stochastic model reduction in multiscale analysis. Prob Engr Mech 2008;197:3584-92. doi:10.1016/j.cma.2008.03.016

[14] Soize C, Ghanem RG. Reduced chaos decomposition with random coefficients of vector-valued random variables and random fields. Comp Meths Appl Mech Engr 2009;198(21-26):192634. doi:10.1016/j.cma.2008.12.035.

[15] Sarkar A, Benabbou N, Ghanem RG. Domain decomposition of stochastic PDEs: theoretical formulations. Int J Numer Meths Engr 2009;77(5):689-701. doi:10.1002/nme.2431.

[16] Ganis B, Yotov I, Zhong M. A stochastic mortar mixed finite element method for flow in porous media with multiple rock types. SIAM J Sci Comp 2011;33(3):1439-74. doi: $10.1137 / 100790689$.

[17] Le Maître OP, Knio OM, Debusschere BJ, Najm HN, Ghanem RG. A multigrid solver for two-dimensional stochastic diffusion equations. Comp Meths Appl Mech Engr 2003;192(4142):4723-44. doi:10.1016/S0045-7825(03)00457-2.

[18] Cottereau R, Clouteau D, Ben Dhia H, Zaccardi C. A stochastic-deterministic coupling method for continuum mechanics. Comp Meths Appl Mech Engr 2011;200:3280-8. doi: 10.1016/j.cma.2011.07.010.

[19] Chevreuil M, Nouy A, Safatly E. A multiscale method with patch for the solution of stochastic partial differential equations with localized uncertainties. Comp Meths Appl Mech Engr 2013;255:255-74. doi:10.1016/j.cma.2012.12.003

[20] Zaccardi C, Chamoin L, Cottereau R, Ben Dhia H. Error estimation and model adaptation for stochastic-deterministic coupling in the Arlequin framework. Int J Numer Meths Engr 2012;Submitted.

[21] Hou TY, Wu XH. A multiscale finite element method for elliptic problems in composite materials and porous media. J Comp Phys 1997;134(1):169-89. doi:10.1006/jcph.1997.5682.

[22] Hughes TJ. Multiscale phenomena: Green's functions, the Dirichlet-to-Neumann formulation, subgrid scale models, bubbles and the origins of stabilized methods. Comp Meths Appl Mech Engr 1995;127(1-4):387-401. doi:10.1016/00457825(95)00844-9.
[23] Hughes TJ, Feijóo GR, Mazzei L, Quincy JB. The variational multiscale method - a paradigm for computational mechanics. Comp Meths Appl Mech Engr 1998;166(1-2):3-24. doi: 10.1016/S0045-7825(98)00079-6.

[24] Velamur Asokan B, Zabaras N. A stochastic variational multiscale method for diffusion in heterogeneous random media. J Comp Phys 2006;218(2):654-76. doi: 10.1016/j.jcp.2006.02.026.

[25] Xu XF. A multiscale stochastic finite element method on elliptic problems involving uncertainties. Comp Meths Appl Mech Engr 2007;196(25-28):2723-36. doi:10.1016/j.cma.2007.02.002.

[26] Ganapathysubramanian B, Zabaras N. Modeling diffusion in random heterogeneous media: data-driven models, stochastic collocation and the variational multiscale method. J Comp Phys 2007;226(1):326-53. doi:10.1016/j.jcp.2007.04.009.

[27] Chen Z, Savchuk TY. Analysis of the multiscale finite element method for nonlinear and random homogenization problems. SIAM J Numer Anal 2008;46(1):260-79. doi: $10.1137 / 060654207$.

[28] Velamur Asokan B, Zabaras N. Variational multiscale stabilized FEM formulations for transport equations: stochastic advection-diffusion and incompressible stochastic NavierStokes equations. J Comp Phys 2005;202(1):94-133. doi: 10.1016/j.jcp.2004.06.019.

[29] Ganapathysubramanian B, Zabaras N. A stochastic multiscale framework for modeling flow through random heterogeneous porous media. J Comp Phys 2009;228(2):591-618. doi: 10.1016/j.jcp.2008.10.006

[30] Jiang L, Mishev I, Li Y. Stochastic mixed multiscale finite element methods and their applications in random porous media. Comp Meths Appl Mech Engr 2010;199(41-44):2721-40. doi: 10.1016/j.cma.2010.05.018.

[31] Ginting V, Malqvist A, Presho M. A novel method for solving multiscale elliptic problems with randomly perturbed data. SIAM Multiscale Model Simul 2010;8(3):977-96. doi: 10.1137/090771302.

[32] Le Bris C, Legoll F, Thomines F. Multiscale finite element approach for "weakly" random problems and related issues. arXiv:1111.1524v1; 2011.

[33] Aarnes JE, Efendiev Y. Mixed multiscale finite element methods for stochastic porous media flows. SIAM J Sci Comp 2008;30(5):2319-39. doi:10.1137/07070108X.

[34] Dostert P, Efendiev Y, Hou TY. Multiscale finite element methods for stochastic porous media flow equations and applications to uncertainty quantification. Comp Meths Appl Mech Engr 2008;197(43-44):3445-55. doi:10.1016/j.cma.2008.02.030.

[35] Jiang L, Presho M. A resourceful splitting technique with application to deterministic and stochastic multiscale finite element methods. SIAM Multiscale Model Simul 2012;10(3):954-85. doi:10.1137/110843253.

[36] E W, Ming P, Zhang P. Analysis of the heterogeneous multiscale method for elliptic homogenization problems. J Amer Math Soc 2005;18:121-56. doi:10.1090/S0894-0347-0400469-2.

[37] Ma X, Zabaras N. A stochastic mixed finite element heterogeneous multiscale method for flow in porous media. J Comp Phys 2011;230(12):4696-722. doi:10.1016/j.jcp.2011.03.001.

[38] Bal G, Jing W. Corrector theory for MsFEM and HMM in random media. SIAM Multiscale Model Simul 2011;9(4):1549-87. doi: $10.1137 / 100815918$.

[39] Stein E, Ohnimus S. Coupled model- and solution-adaptivity in the finite-element method. Comp Meths Appl Mech Engr 1997;150(1-4):327-50. doi:10.1016/S0045-7825(97)00082-0.

[40] Melenk JM, Babuška I. The partition of unity finite element method: basic theory and applications. Comp Meths 
Appl Mech Engr 1996;139(1-4):289-314. doi:10.1016/S00457825(96)01087-0.

[41] Moës N, Dolbow J, Belytschko T. A finite element method for crack growth without remeshing. Int $\mathrm{J} \mathrm{Nu}$ mer Meths Engr 1999;46(1):131-50. doi:10.1002/(SICI)10970207(19990910)46:1;131::AID-NME726;3.0.CO;2-J.

[42] Düster A, Niggl A, Rank E. Applying the hp-d version of the FEM to locally enhance dimensionally reduced models. Comp Meths Appl Mech Engr 2007;196(37-40):3524-33. doi: 10.1016/j.cma.2006.10.018.

[43] Gendre L, Allix O, Gosselet P. A two-scale approximation of the Schur complement and its use for non-intrusive coupling. Int J Numer Meths Engr 2011;87(9):889-905. doi: 10.1002/nme.3142.

[44] Lozinski A, Pironneau O. Numerical zoom for advection diffusion problems with localized multiscales. Numer Meth Part Diff Equ 2011;27(1):197-207. doi:10.1002/num.20642.

[45] Hager C, Hauret P, Le Tallec P, Wohlmuth BI. Solving dynamic contact problems with local refinement in space and time. Comp Meths Appl Mech Engr 2012;201-204:25-41. doi: 10.1016/j.cma.2011.09.006.

[46] Ben Dhia H. Multiscale mechanical problems: the Arlequin method. Comptes Rendus Acad Sci - Series IIB - MechPhys-Astron 1998;326(12):899-904. doi:10.1016/S12518069(99)80046-5.

[47] Brezzi F, Lions JL, Pironneau O. Analysis of a Chimera method. Comptes Rendus Acad Sci - Series I - Math 2001;332(7):65560. doi:10.1016/S0764-4442(01)01904-8.

[48] Wagner GJ, Liu WK. Coupling of atomistic and continuum simulations using a bridging scale decomposition. J Comp Phys 2003;190:249-74. doi:10.1016/S0021-9991(03)00273-0.

[49] Xiao SP, Belytschko T. A bridging domain method for coupling continua with molecular dynamics. Comp Meths Appl Mech Engr 2004;193(17-20):1645-69. doi: 10.1016/j.cma.2003.12.053.

[50] Cottereau R, Ben Dhia H, Clouteau D. Localized modeling of uncertainty in the Arlequin framework. In: Langley R, Belyaev A, editors. Vibration Analysis of Structures with Uncertainties. IUTAM Bookseries; Springer; 2010, p. 477-88. doi: 10.1007/978-94-007-0289-9_33.

[51] Chamoin L, Oden JT, Prudhomme S. A stochastic coupling method for atomic-to-continuum Monte-Carlo simulations. Comp Meths Appl Mech Engr 2008;197(43-44):3530-46. doi:10.1016/j.cma.2008.04.013.

[52] Babuška I, Tempone R, Zouraris GE. Galerkin finite element aproximations of stochastic elliptic partial differential equations. SIAM J Numer Anal 2004;42(2):800-25. doi: $10.1137 /$ S0036142902418680.

[53] Ghanem RG, Spanos PD. Stochastic finite elements: a spectral approach. Springer-Verlag; 1991.

[54] Stefanou G. The stochastic finite element method: past, present and future. Comp Meths Appl Mech Engr 2009;198(912):1031-51. doi:10.1016/j.cma.2008.11.007.

[55] Robert CP, Casella G. Monte Carlo statistical methods. Springer; 2004. ISBN 0387212396.

[56] Papanicolaou GC, Varadhan SR. Boundary value problems with rapidly oscillating random coefficients. In: Fritz J, Lebowitz JL, editors. Proceedings of the Conference on Random Fields; vol. 2 of Seria Colloquia Mathematica Societatis Janos Bolyai. North Holland; 1981, p. 835-73.

[57] Bourgeat A, Piatnitski A. Approximations of effective coefficients in stochastic homogenization. Ann Inst Henri Poincaré 2004:40:153-65. doi:10.1016/j.anihpb.2003.07.003.

[58] Tartar L. The general theory of homogenization: a personalized introduction; vol. 7 of Lecture notes of the Unione Matematica
Italiana. Springer; 2009.

[59] Cottereau R. Numerical strategy for unbiased homogenization of random media. Int J Numer Meths Engr 2013;Accepted for publication.

[60] Nœtinger B, Artus V, Zargar G. The future of stochastic and upscaling methods in hydrogeology. Hydrogeol J 2005;13:184201. doi:10.1007/s10040-004-0427-0.

[61] Milton GW. The theory of composites. Cambridge Monographs on Applied and Computational Mechanics; Cambridge University Press; 2002.

[62] Wen XH, Gómez-Hernández J. Upscaling hydraulic conductivities in heterogeneous media: an overview. J Hydrology 1996;183(1-2):ix-xxxii. doi:10.1016/S0022-1694(96)80030-8.

[63] Renard P, de Marsily G. Calculating equivalent permeability: a review. Adv Water Resource 1997;20(5-6):253-78. doi: 10.1016/S0309-1708(96)00050-4.

[64] Farmer CL. Upscaling: a review. Int J Numer Meth Fluids 2002;40(1-2):63-78. doi:10.1002/fld.267.

[65] Brewster ME, Beylkin G. A multiresolution strategy for numerical homogenization. Appl Comp Harmonic Anal 1995;2(4):327-49. doi:10.1006/acha.1995.1024.

[66] Dorobantu M, Engquist B. Wavelet-based numerical homogenization. SIAM J Numer Anal 1998;35(2):540-59. doi: 10.1137/S0036142996298880.

[67] Ben Dhia H, Rateau G. Mathematical analysis of the mixed Arlequin method. Comptes Rendus Acad Sci - Series I - Math 2001;332(7):649-54. doi:10.1016/S0764-4442(01)01900-0.

[68] Ben Dhia H, Rateau G. The Arlequin method as a flexible engineering design tool. Int J Numer Meths Engr 2005;62(11):144262. doi:10.1002/nme.1229.

[69] Ben Dhia H. Further insights by theoretical investigations of the multiscale Arlequin method. Int J Multiscale Comp Engr 2008;6(3):215-32. doi:10.1615/IntJMultCompEng.v6.i3.30. 